\title{
The effect of strabismus on object detection in the ring scotoma of a monocular bioptic telescope
}

\author{
Amy L. Doherty, Alex R. Bowers, Gang Luo, and Eli Peli \\ Schepens Eye Research Institute, Mass Eye and Ear, Harvard Medical School, Boston, MA
}

\begin{abstract}
Purpose-People with reduced visual acuity are permitted to drive with the aid of bioptic telescopes in the USA, The Netherlands, and Canada. When viewing through a monocular bioptic telescope, suppression of the deviating eye in strabismus may reduce the ability of the nontelescope eye to detect objects whose images fall in the ring scotoma area of the telescope eye, which could impair detection of traffic-relevant events. This ability to detect stimuli in the ring scotoma area was compared for strabismic and non-strabismic patients.
\end{abstract}

Methods-Ten strabismic and six non-strabismic patients with bilaterally reduced visual acuity (6/12 to 6/60) participated. A dichoptic perimeter presented stimuli to the fellow (non telescope) eye in the area of the ring scotoma under binocular viewing. Fellow-eye detection rates were determined with and without a bioptic, on uniform and patterned backgrounds, while performing passive (viewing a cross) and active (reading letters) fixation tasks.

Results-All strabismic patients were found to have anomalous retinal correspondence. Both non-strabismic and strabismic patients had lower fellow-eye detection rates on patterned than on uniform backgrounds, and while performing the active task. In addition, strabismic patients had lower detection with than without the bioptic on the patterned background. They also had a larger decrease in detection from the uniform to the patterned background than non-strabismic patients ( $26 \%$ vs $8 \%$ ). Depending on the angle and direction of the deviation relative to the target side, strabismus either increased or decreased fellow-eye stimulus eccentricity on the retina. Larger detection rate reductions between the uniform and patterned backgrounds were associated with more eccentric stimulus locations ( $\mathrm{rho}=0.606, \mathrm{p}=0.013$ ).

Conclusions-Both strabismic patients and non-strabismic patients were able to detect stimuli with the fellow eye in the ring scotoma area, demonstrating successful bi-ocular multiplexing. However, strabismic patients generally had a greater reduction in detection performance from the uniform to the patterned background than non-strabismic patients, which was accounted for in part by differences in stimulus eccentricities on the retina (that varied with the angle and direction of the strabismus). However, a study with a larger sample, including participants with strabismus and normal retinal correspondence, is needed before our findings can be generalized.

\section{Keywords}

low vision; rehabilitation; vision multiplexing; driving

\section{Introduction}

Bioptic telescopes are small, spectacle-mounted telescopes that enable people with reduced visual acuity to resolve details of distant objects such as road signs. ${ }^{1}$ They are permitted as a 
visual aid for driving in up to 43 US states, ${ }^{2,}{ }^{3}$ the Netherlands, ${ }^{4}$ and Quebec, Canada. The telescope is usually mounted in the upper part of the lens. Most of the time when driving, the wearer views through the carrier lens (below the telescope). When a detailed view of a distant object is needed, the wearer tilts his head down and looks briefly through the telescope. The majority of bioptic drivers are prescribed a monocular telescope, presumably to enable continued surveillance of the road by the fellow (non-telescope eye). ${ }^{1,5,6}$ In the Netherlands, ${ }^{4}$ and at least in Massachusetts, USA, ${ }^{7}$ only monocular telescopes are allowed for driving.

When using a telescope, the magnified field of view on the retina covers much of the image normally available in the unmagnified view. This creates a 'ring scotoma' around the magnified telescope view. When viewing natural scenes the difference in image size between the monocular telescope eye and fellow eye may cause binocular rivalry, as two different images fall on corresponding retinal points. In binocular rivalry, perception alternates between the two images (or parts of the images) with one dominant while the other is suppressed, and then the roles are reversed. ${ }^{8}$ The predominant image is the one that dominates more often during the rivalry and is perceived for a greater portion of time. Many factors may affect predominance. When one eye's image is more salient in terms of brightness, contrast, details, motion velocity, or color, it will predominate. Attention to one eye's image can also increase that eye's predominance. ${ }^{9-11}$ Since attention is directed at the telescope image during active viewing, and that image is likely to be more detailed, the image seen by the fellow non-telescope eye is less likely to predominate. In the extreme case, the fellow non-telescope eye might even be persistently suppressed. Thus detection of traffic-relevant events could be affected if they happen to fall within the ring scotoma, the area in the scene that is visible only to the fellow eye. Indeed, some have argued that for this reason, the ring scotoma makes bioptic driving dangerous. ${ }^{12-14}$

In our previous study of (non-strabismic) patients fitted with monocular bioptic telescopes, we found that the fellow non-telescope eye was able to detect stimuli presented in the area of the ring scotoma. ${ }^{15} \mathrm{We}$ found no evidence of reduced fellow-eye predominance or suppression when the telescope was used; but would the same be true for patients with strabismus? This is an important question to address, as about $60 \%$ of bioptic drivers have congenital ocular conditions, such as albinism and nystagmus, $1,5,6,16$ where strabismus is a common co-morbidity. ${ }^{17-20}$

In strabismus, monocular bioptic telescopes are typically fitted in front of the dominant, non-deviating eye. As strabismus is associated with strong ocular dominance, fitting the bioptic on the dominant eye could decrease predominance ${ }^{21}$ of the non-dominant fellow eye during binocular rivalry, or even result in persistent suppression of the fellow eye.

Suppression (whole or partial/local) occurring under binocular viewing conditions prevents diplopia. However, in this case it could reduce detection in the area of the fellow eye corresponding to the ring scotoma. In contrast to suppression, anomalous retinal correspondence (ARC) is also a common sensory adaptation in patients with congenital (or early childhood onset) strabismus ${ }^{22,23}$ that prevents diplopia without requiring suppression. Therefore, it is possible that strabismic patients with ARC are better able to detect objects in the ring scotoma than those who retain normal correspondence and adapt by suppressing the deviating eye.

The retinal eccentricity of an object imaged by the deviating eye varies with the angle of deviation, which may affect both predominance of the deviating eye ${ }^{24}$ and object (e.g., pedestrian) visibility. ${ }^{25}$ Depending on the placement of the object relative to the fovea of the non-dominant (deviating) eye, the retinal eccentricity of the object can be greater or smaller than the eccentricity of the same object in the fellow eye of a non-strabismic patient. For 
example, for a left esotrope, objects appearing on the left side of the ring scotoma would fall on a more eccentric retinal area of the deviating eye than in a non-strabismic patient (Figure 1). Increased eccentricity may reduce performance due to the decreased sensitivity of peripheral retina ${ }^{25-27}$ as well as increased effects of crowding with increasing eccentricity. ${ }^{28}$ Furthermore, the likelihood of one image being persistently suppressed during binocular rivalry increases with increasing retinal eccentricity. ${ }^{24}$ On the other hand, it is also possible for stimuli to fall on more central retina, for example if objects appear on the right side of the ring scotoma in a left esotrope (Figure 1). If eccentricity is an important factor, then detection of objects should either improve or decline as eccentricity is closer to or farther from the fovea of the deviating eye, respectively (Figure 1). Conversely, images falling on more central retina could also be suppressed, as the fovea of the deviating eye is often suppressed. ${ }^{29,}{ }^{30}$ However, as mentioned above, suppression is less likely in patients who develop ARC. Nevertheless, ARC can break down to suppression under unnatural viewing conditions, and even if ARC is maintained, there is usually a suppression scotoma at both the fovea of the deviating eye and the retinal location corresponding to the fovea of the nondeviating eye.

In this study we evaluated the ability of patients with strabismus to use the fellow (nontelescope) eye to detect targets while viewing through a monocular bioptic telescope. Specifically, we compared detection performance of strabismic and non-strabismic patients with bilaterally reduced visual acuity using the same methodology as our previous study. ${ }^{15}$ Based on our prior findings, ${ }^{15}$ we expected to find lower fellow-eye detection rates on the patterned than the uniform background and in the active than passive viewing task for both strabismic and non-strabismic patients in this study. Importantly, we also predicted that there would be an additional effect of strabismus. Specifically, that fellow-eye detection rates would be more reduced in strabismic than non-strabismic patients on the patterned background, especially when using the bioptic, because of the increased likelihood of suppression in strabismus on complex backgrounds particularly for patients who have not developed ARC. In addition, we examined the effects of stimulus retinal eccentricity on fellow-eye detection rates.

\section{Methods}

\section{Participants}

Thirteen patients with reduced visual acuity (VA) and strabismus were recruited from local clinics and the National Organization on Albinism and Hypopigmentation. Inclusion criteria were VA of $6 / 12$ to $6 / 60$ in the better-seeing (telescope) eye and no central visual field loss. Three of the 13 recruited patients did not complete the study (failed to attend the second visit for non visual reasons) and their data were excluded. In addition, data from 6 patients with reduced VA but without strabismus were included for comparison purposes. Participants in the comparison group had no central visual field loss and were selected to provide a similar distribution of ages and visual acuities as in the strabismic group. (Data for five of these participants were from our previous study ${ }^{15}$ ). Monocular and binocular single letter visual acuity (TestChartPro2000, Thomson Software Solutions, Potters Bar, Hertfordshire, UK) was measured with and without the bioptic used for the study. The presence (cover-uncover test) and magnitude of strabismus (alternating cover test) were determined using an accommodative target large enough for the patient to read with either eye at $1 \mathrm{~m}$ (the viewing distance used for the detection tests). The Bagolini Striated Lens test was used to measure central retinal correspondence ${ }^{31,32}$ in a darkened room (so participants could distinguish the streaks despite their reduced acuity). Suppression was examined for all participants using the Worth Four Dot test. Strabismic participants were also evaluated with the suppression test of the Randot Stereo Test (Stereo Optical Company Inc., Chicago IL, 
USA). Fourteen of the sixteen participants had fixation stability measured using the Nidek MP-1 microperimeter (NIDEK Co, Ltd, Gamagori, Japan) while they monocularly fixated a $2^{\circ}$ cross for $30 \mathrm{~s}$.

Eight of the 16 participants (6 strabismic and 2 non-strabismic) owned and had used a bioptic telescope within the past year. The remaining participants had never used a bioptic. In our previous study, there were no significant differences in detection rates between bioptic users and non-users. A short survey was used to quantify bioptic usage.

The study was conducted in accordance with the tenets of the Declaration of Helsinki. All participants signed a consent form approved by the Schepens Eye Research Institute Institutional Review Board.

\section{Bioptic telescopes}

Patients were fitted with either a 3.0x Keplerian (Mini, Ocutech Inc., Chapel Hill, NC) or 3.0x Galilean (Designs for Vision (DVI), Inc., Ronkonkoma, NY) bioptic telescope on their sighting-dominant eye (determined using the hole-in-the-card test ${ }^{33}$ ). Eight patients used the Keplerian telescope (adjusting the focus for $1 \mathrm{~m}$ ), 5 used the Galilean (with a $+1.00 \mathrm{D}$ reading cap), and 3 patients used their own focusable 3.0x Keplerian Mini bioptic for the study (one to correct for high astigmatism and two because the bioptics were positioned and angled for the nystagmus null point ${ }^{34}$ ). The telescopes provided by us were mounted centrally in the carrier lens of an adjustable bridge frame, not in the bioptic position, in order to conduct the experiment without the added strain of maintaining a head tilt and eccentric gaze position. A single-vision lens with the patient's habitual distance prescription including an additional $+1.00 \mathrm{D}$ was fitted for the fellow eye.

\section{Apparatus}

A custom, dichoptic perimetry system ${ }^{35}$ was used to enable separate presentation of stimuli to each eye. With this system, the fixation target and background view could be presented to both eyes while the stimulus was presented to only one eye. This assured that the stimulus was not seen by the telescope eye, even if the ring scotoma had shifted due to head or eye movements.

Participants viewed through ferroelectric liquid crystal shutter lenses (Cambridge Research System, Rochester, Kent, England) used with an InFocus DepthQ projector (Lightspeed Design Group, Bellevue, Washington, http://www.depthq.com) run at 120Hz. For detection tests, participants wore a lightweight modified indirect ophthalmoscope headband with the shutter lenses suspended in front of the eyes to provide sufficient clearance for the telescope. ${ }^{15}$ The $6.5 \mathrm{~cm}$ distance between the shutters and the eyes restricted the field of view to about $25^{\circ}$ diameter, with the visible area extending farther temporally than nasally in each eye, especially for participants with narrow interpupillary distances (interpupillary distance of the shutter lenses was fixed at $68 \mathrm{~mm}$ ). This restriction limited the area available for fellow-eye stimulus presentation to the temporal side of that eye (see Procedure for more detail).

\section{Detection test conditions}

The two backgrounds implemented in our previous study ${ }^{15}$ were used: uniform gray (level 186 out of 255) and a patterned spatial noise background which changed after each trial (Figure 2). The patterned background had spatial frequency characteristics similar to natural scenes ${ }^{36}$ and has been shown to cause binocular rivalry when viewing through a monocular bioptic telescope. ${ }^{15}$ The patterned background was used rather than natural images to control for the varying contrast, luminance and clutter in real-world pictures that could 
affect target detection differently in different presentations and locations. Mean luminance of all backgrounds was $38 \mathrm{~cd} / \mathrm{m}^{2}\left(8 \mathrm{~cd} / \mathrm{m}^{2}\right.$ through the shutter lenses in the open state).

Two types of fixation tasks, identical to those in our previous study, ${ }^{15}$ were used (Figure 2): a passive task looking at a bipolar cross $\left(2.9^{\circ}\right)$ and an active task reading letters $\left(0.7^{\circ}\right.$ or $1.0^{\circ}$ with the bioptic, $1.8^{\circ}$ or $2.2^{\circ}$ without the bioptic). In the reading task, single black letters inside a white box were presented at a comfortable reading size for participants, with letters changing every 2 seconds. For all but one of the patients, the test stimuli were $1.0^{\circ}$ square $2 \times 2$ black and white checkerboards with an internal Michelson contrast of $75 \%$ or $95 \%$. The contrast level was selected for each patient based on pilot trials to avoid ceiling (100\% detection) and floor (0\% detection) effects on the patterned background, and then used consistently for each patient in all trials. Five patients (4 strabismic and 1 non-strabismic) were presented with the $95 \%$ contrast stimulus; the $75 \%$ contrast stimulus was used for the others. One strabismic patient (with near floor effects with the $1.0^{\circ}$ stimulus) was presented a $1.4^{\circ}$ square stimulus with a contrast of $50 \%$ (to avoid near ceiling effects with the $75 \%$ contrast stimulus). Stimuli were presented for $250 \mathrm{~ms}$. Participants were given a grace period of $600 \mathrm{~ms}$ after stimulus presentation to respond. To avoid predictability, a randomized delay between $1000 \mathrm{~ms}$ and $1950 \mathrm{~ms}$ occurred before the onset of the next stimulus.

\section{Procedure}

At the start of each session, the field of view through the bioptic telescope and the outer boundary of the ring scotoma were measured under monocular viewing conditions with the fellow eye patched (Figure 3A). Participants then performed a series of 8 test conditions with dichoptic presentations in a $2 \times 2 \times 2$ design (with and without bioptic, with passive or active fixation task, on uniform gray or patterned background). Testing was divided into 2 blocks of 4 conditions, with the order of fixation task and background counterbalanced within each block and across patients. Half used the bioptic for the first 2 conditions of each block; however, counterbalancing was not complete as there were 16 participants (and 24 possible orderings for a block). The total time to complete the 8 conditions was approximately 1 hour.

Background and fixation targets were presented to both eyes. Stimuli were presented to either the telescope or fellow eye only. The field of view of the fellow eye through the shutters was measured perimetrically by presenting the stimulus to the fellow eye only under binocular viewing (Figure 3B). Thirteen random locations were selected manually to present stimuli within the area corresponding to the ring scotoma that was within the field of view of the fellow eye through the shutters. Due to the asymmetry of the area visible to the fellow eye (wider temporally than nasally), stimuli were only presented in the temporal field of the fellow-eye (Figure 3C). Thus for patients with esotropia, stimuli eccentricities were more eccentric than for non-strabismics. For exotropes, stimuli eccentricities were closer to the fovea of the deviating eye and either similar to or more central than for non-strabismics. Thus, the retinal locations of the 13 stimuli varied across participants.

Each condition contained 5 blocked repetitions of 13 stimulus locations, with the order of stimulus positions randomly changed for each repetition. Of the 65 total stimuli, 60 were presented to the fellow eye only, and 5 were presented to the telescope eye only, as catch trials to monitor fixation (as they should not have been seen by the telescope eye). In general fixation was good: across all the bioptic conditions, 6 participants did not respond to any of these catch stimuli, and the remaining 10 participants responded to just 1 (median) out of the total 20 catch trials, with the maximum number of catch-trial responses being 4 .

Participants were instructed to press a hand-held response button when a stimulus was detected while fixating a central cross in the passive task or verbally reporting each letter in 
the active task. The active task ensured that fixation and attention were maintained. For blocks in which the telescope was used, fixation was through the telescope.

\section{Data Analysis}

Detection performance (number of detections as a fraction of the total number of stimulus presentations) for each test condition was the primary dependent variable. Percentiles were converted to $\mathrm{z}$ scores by a probit transform to avoid the truncation effect ${ }^{37,38}$ and also improve the normality of the data. A value of 2.4 was used in cases where $100 \%$ of stimuli were detected and -2.4 in cases where no stimuli were detected. Data was analyzed using a repeated measures analysis of variance (ANOVA). There were three within-subjects factors: bioptic (with/without), fixation task (passive/active) and background (uniform/patterned). Group (strabismic/non-strabismic) was the between-subjects factor. Stimulus contrast varied across participants and was, therefore, included as a covariate in the analysis. The findings were similar with and without the covariate; only results for the ANOVA without the covariate are reported. For ease of interpretation, z scores were converted back to percentiles and fellow-eye detection results are presented as percentiles in the results.

The retinal eccentricity of each stimulus presented to the fellow eye was calculated by computing the angular distance between the direction of the deviated fovea (the angle of deviation from the cover test at $1 \mathrm{~m}$ ) and each stimulus location. The median eccentricity for the 12 stimulus locations presented to the fellow eye (in degrees) was used in analyses. Spearman's correlation coefficients were used to quantify the relationship between stimulus retinal eccentricity and the difference in fellow-eye detection rates between the uniform and patterned backgrounds. The difference in detection rates (rather than the absolute patternedbackground detection rate) was used in order to normalize the data and remove the effects of between-subject variability in "baseline" detection performance.

\section{Results}

\section{Sample Characteristics and Bioptic Usage}

Participants had a range of congenital visual conditions; 7 of 16 had albinism and 13 had nystagmus (Table 1). There was no significant difference in age, visual acuity or contrast sensitivity between strabismic and non-strabismic patients (Table 2). Of the 10 patients with strabismus, seven were exotropes ( 3 right, 1 left, 3 alternating) and 3 were esotropes ( 1 right, 1 left, 1 alternating) with a median angle of strabismus of $22 \Delta$ All exhibited ARC on the Bagolini Striated Lens test in a darkened room, conditions under which ARC is less likely to be demonstrated than in normal ambient room illumination. All strabismic and 2 nonstrabismic patients showed diplopia on the Worth Four Dot test, 3 non-strabismics showed fusion, and 1 suppressed the non-dominant eye. On the Randot stereo suppression test, 6 strabismic patients alternated between eyes, 1 suppressed the non-dominant eye, and 3 reported both eyes' stimuli were present simultaneously with the non-dominant eye's image dimmer or fading. Two strabismic patients had amblyopia (difference of at least 2 lines in visual acuity); one with better acuity in the telescope eye and the other with better acuity in the fellow eye (telescope was used with the sighting-dominant eye). As expected, fixation stability of patients with nystagmus was poorer than that of patients without nystagmus. However, the vast majority of fixation samples recorded during the fixation stability measurement fell within $4^{\circ}$ of the fixation target in the microperimeter (Table 3 ).

Of the 8 patients ( 6 strabismic and 2 non-strabismic) who had used a bioptic telescope within the past year, time owning the bioptic ranged from 1 month to 37 years with a median time of 6 years. Three patients used the bioptic weekly, 2 monthly, and 2 had not used it in many months. The patient having the bioptic for only 1 month did not complete the survey. 
All had used the bioptic for driving (primarily for reading signs, viewing traffic signals, or spotting potential hazards ahead) and 2 patients additionally used the bioptic when spectators at sporting or theater events. They all reported the telescope as at least moderately helpful on a 5-point scale ranging from 1 (not at all) to 5 (very helpful), with 5 of 7 reporting it very helpful.

\section{Fellow-eye detection ability}

Results of the repeated measures analysis of variance are reported in Table 4. Consistent with our prior study, there were significant reductions in fellow-eye detection rates from $96 \%$ on the uniform background to $80 \%$ on the patterned background (means pooled across bioptic, task and vision group; Figure 4), and from $93 \%$ in the passive task to $87 \%$ in the active task (means pooled across bioptic, background and vision group; Figure 5), but no significant difference in detection rates between viewing with (89\%) and without (92\%) a bioptic. Although there was no significant difference in detection rates between strabismic $(88 \%)$ and non-strabismic (92\%) patients (means pooled across all conditions), there was a significant interaction between background and strabismus $(\mathrm{p}=0.036)$. As Figure 4 shows, strabismic patients had a greater reduction in detection performance from the uniform to the patterned background (26\%: $97 \%$ to $71 \%$ ) than non-strabismic patients (8\%: $95 \%$ to $87 \%$; $\mathrm{p}=0.036$ ), and there was much greater between-subject variability in the strabismic than the non-strabismic group.

To further investigate the effect of the bioptic on detection rates on the patterned background, paired (planned) comparisons of detection rates with and without the bioptic were conducted. Strabismic patients had significantly lower detection rates on the patterned background with than without the bioptic $\left(\mathrm{t}_{(9)}=2.87, \mathrm{p}=0.018\right.$; Figure $\left.6 \mathrm{~A}\right)$, but nonstrabismic patients did not $\left(\mathrm{t}_{(5)}=-0.318, \mathrm{p}=0.764\right)$. By comparison, on the uniform background, the strabismic and non-strabismic groups behaved in a similar fashion with no significant differences in detection rates with and without the bioptic (Figure 6B), though there might have been a ceiling effect in this case. The non-strabismic patient that suppressed the non-dominant eye on the Worth Four Dot Test was the only patient in the non-strabismic group to have lower detection rates with than without the bioptic on the patterned background (Figure 6A).

\section{Fellow-eye stimulus eccentricity and detection rates}

As detailed in the procedures, fellow-eye stimulus retinal eccentricity was affected by the type of strabismus (eso/exo), whether it was the left or right eye that deviated, and the magnitude of the deviation. Furthermore, due to the limitations imposed by the shutter lenses' fixed interpupillary distance, we were able to place stimuli in only the temporal field of the fellow eye. As a result, for four of the strabismic patients, the stimulus eccentricities were much larger $\left(>15^{\circ}\right)$ than for the non-strabismic patients $\left(7^{\circ}-10^{\circ}\right)$, for five they were about the same, and for one patient they were slightly lower (Figure 7). Overall, however, larger stimulus retinal eccentricities were associated with larger angle deviations (Figure 7).

Stimulus retinal eccentricity was positively correlated with the difference in detection rates between the uniform and patterned backgrounds. Patients with stimuli presented at larger fellow-eye retinal eccentricities tended to have a larger reduction in detection rate than patients with stimuli presented at smaller eccentricities (Figure 8). This was true when data were analyzed separately for the with- and without-bioptic conditions (rho $=0.579 \mathrm{p}=$ 0.019 ; rho $=0.544 \mathrm{p}=0.029$, respectively) or pooled across these conditions (Figure 8 , rho $=0.603, \mathrm{p}=0.013$ ). 
It is notable that there were two strabismic patients (circled symbols) with only a small difference in detection rates between the uniform and patterned backgrounds (Figure 8), differences similar to the smallest difference in the non-strabismus group. Stimulus eccentricity for these two patients was closer to the fellow-eye fovea than for other strabismic patients (Figure 7).

\section{Vision measures and fellow-eye detection rates}

Neither fellow-eye visual acuity nor binocular contrast sensitivity correlated with detection rates on the uniform background, patterned background or the difference in detection rates between the two backgrounds ( $\mathrm{n}=16$, Spearman's rho $<0.328, \mathrm{p}>0.214)$.

\section{Discussion}

Both strabismic and non-strabismic patients were able to detect stimuli with the fellow eye in the ring scotoma area while viewing through a monocular bioptic telescope, exhibiting successful bi-ocular multiplexing. ${ }^{39}$ Consistent with our previous study, ${ }^{15}$ we found an overall reduction in fellow-eye detection performance under the more complex viewing conditions, on the patterned background and in the active viewing task. However, strabismic patients had significantly lower detection rates with than without the bioptic on the patterned background (Figure 6A) but not the uniform background (Figure 6B), while non-strabismic patients showed no differences in detection rates with and without the bioptic on both the patterned and uniform backgrounds. Although strabismic patients did show bi-ocular multiplexing, their detection performance was more likely to decrease than the nonstrabismic patients when stimuli were presented over patterned backgrounds. This suggests that partial suppression of the fellow eye (through rivalry-increased predominance of the telescope eye) could manifest in more real-world situations for bioptic drivers with strabismus and ARC.

The reduction in detection rates from the uniform to the patterned backgrounds was greater for strabismic than non-strabismic patients. Patients whose stimuli fell on more eccentric retinal areas had larger reductions in detection rates. The two strabismic patients with stimuli presented more centrally than most non-strabismic patients showed the smallest reductions in detection rates from the uniform to the patterned backgrounds (Figure 8), suggesting that the effect might have more to do with stimulus retinal eccentricity than with the presence of strabismus per se in patients with ARC.

The majority of participants had nystagmus, a common condition among bioptic drivers. ${ }^{1,5,6,16}$ While the nystagmus may appear to continuously vary the retinal eccentricity of stimuli, vision takes place mostly during the short fixation periods that occur within the typical nystagmic eye movement pattern of people with infantile nystagmus syndrome. ${ }^{40}$ The fixation stability of patients with nystagmus was indeed poorer than that of patients without nystagmus; however, the majority of fixation samples were within $4^{\circ}$ of the fixation target when evaluated in a microperimeter. Seven of the patients in our study had albinism. In albinism the routing of nerve fibers in the chiasm is abnormal, ${ }^{18}$ resulting in abnormal binocular vision. The nature of this binocular vision is not well understood and requires further investigation. We are pursuing such studies, in particular in relation to the high prevalence of ARC found in our study population. As varied as our population was, all the strabismic patients exhibited ARC and thus we did not have an opportunity to evaluate the effects of strabismus with normal retinal correspondence and potential suppression on fellow-eye detection rates when using a bioptic.

To fully examine the effect of eccentricity and strabismus on detection in the ring scotoma area, further testing with stimuli presented in a wider area, and on both sides of the ring 
scotoma is needed. While strabismus increases the eccentricity on the fellow-eye retina of stimuli that fall on one side of the ring scotoma of the bioptic eye, it typically decreases the eccentricity of stimuli on the other side (except for very large angle deviations). In general, therefore, the performance decrement on one side may be accompanied by a performance increment on the other side. The relative value of better detection performance on the right or left when driving is not known and may depend on the side of driving (on the left, as in the UK, vs. on right in the USA) ${ }^{41}$ However, at a minimum, patients should be advised about the potential difference in their detection ability on each side, though it is not clear how they might attempt to compensate for this difference.

Taken together, the findings presented in our previous paper ${ }^{15}$ and this one suggest that a standard perimetric evaluation (as used in some prior bioptic telescope studies ${ }^{42}$ ) is insufficient to determine the effect of the ring scotoma on detection ability and highlight the importance of using testing conditions that resemble real world use of devices. Performing perimetry using a patterned background and while engaged in an attention-demanding central task, fellow-eye detection performance in the area corresponding to the ring scotoma was reduced relative to performance in conditions more representative of conventional perimetry (uniform background and passive viewing of a central fixation cross). This was true for both strabismic patients with ARC and non-strabismic patients. However, the reduction was generally greater for the strabismic patients than for the non-strabismic patients, because for most of them the stimulus retinal eccentricity was larger. On the other side of the ring scotoma the eccentricity is usually lower; therefore, the difference between strabismic patients with ARC and non-strabismic patients might have been less if we had been able to measure fellow-eye detection performance on both sides of the ring scotoma. However, a study with a larger sample size is needed before firm conclusions can be drawn. We are conducting further studies to evaluate the ability to detect realistic targets in more naturalistic conditions involving motion backgrounds (driving videos). ${ }^{43}$

\section{Acknowledgments}

Supported in part by grants: EY018680 (A. Bowers), AG034553 (G. Luo), and EY12890 (E. Peli). Premnandhini Satgunam conducted binocular vision tests.

\section{References}

1. Bowers AR, Apfelbaum DH, Peli E. Bioptic telescopes meet the needs of drivers with moderate visual acuity loss. Invest Ophthalmol Vis Sci. 2005; 46(1):66-74. [PubMed: 15623756]

2. Owsley C. Driving with bioptic telescopes: organizing a research agenda. Optom Vis Sci. 2012; 89(9):1249-1256. [PubMed: 22863791]

3. Peli, E.; Peli, D. Driving With Confidence: A Practical Guide to Driving With Low Vision. Singapore: World Scientific; 2002.

4. Melis-Dankers BJM, Kooijman AC, Brouwer WH, et al. A demonstration project on driving with reduced visual acuity and a bioptic telescope system in the Netherlands. Vis Imp Res. 2008; 10:722.

5. Corn AL, Lippmann O, Lewis MC. Licensed drivers with bioptic telescopic spectacles: user profiles and perceptions. Re:view. 1990; 21(4):221-230.

6. Park WL, Unatin J, Park JM. A profile of the demographics, training and driving history of telescopic drivers in the state of Michigan. J Am Optom Assoc. 1995; 66(5):274-280. [PubMed: 7629367]

7. American Medical Association. Physician's Guide to Assessing and Counseling Older Drivers [PDF]. AMA; 2010. Available from: http://www.ama-assn.org/ama1/pub/upload/mm/433/olderdrivers-chapter8.pdf [accessed 1/14/13]

8. Blake R. A primer on binocular rivalry, including current controversies. Brain and Mind. 2001; 2:538. 
9. Chong SC, Tadin D, Blake R. Endogenous attention prolongs dominance durations in binocular rivalry. J Vis. 2005; 5(11):1004-1012. [PubMed: 16441198]

10. Lack L. Selective attention and the control of binocular rivalry. Mouton The Hague. 1978

11. Ooi TL, He ZJ. Binocular rivalry and visual awareness: the role of attention. Perception. 1999; 28(5):551-574. [PubMed: 10664754]

12. Fonda G. Bioptic telescopic spectacle is a hazard for operating a motor vehicle. Arch Ophthalmol. 1983; 101:1907-1908. [PubMed: 6651597]

13. Keeney AH. Field Loss vs. Central Magnification. Arch Ophthalmol. 1974 Oct.92:273. [PubMed: 4412945]

14. Keeney AH, Weiss S, Silva D. Functional problems of telescopic spectacle in the driving task. Trans Am Ophthalmol Soc. 1974; 72:132-138. [PubMed: 4462239]

15. Doherty AL, Bowers AR, Luo G, Peli E. Object detection in the ring scotoma of a monocular bioptic telescope. Arch Ophthalmol. 2011; 129(5):611-617. [PubMed: 21555615]

16. Taylor DG. Telescopic spectacles for driving: user data satisfaction, preferences and effects in vocational, educational and personal tasks: a study in Illinois. J Vis Rehab. 1990; 4(2):29-59.

17. Brodsky MC, Fray KJ. The prevalence of strabismus in congenital nystagmus: the influence of anterior visual pathway disease. J AAPOS. 1997; 1(1):16-19. [PubMed: 10530980]

18. Gronskov K, Ek J, Brondum-Nielsen K. Oculocutaneous albinism. Orphanet J Rare Dis. 2007; 2:43. [PubMed: 17980020]

19. Kinnear PE, Jay B, Witkop CJ Jr. Albinism. Surv Ophthalmol. 1985; 30(2):75-101. [PubMed: 3934778]

20. Kumar A, Gottlob I, McLean RJ, Thomas S, Thomas MG, Proudlock FA. Clinical and oculomotor characteristics of albinism compared to FRMD7 associated infantile nystagmus. Invest Ophthalmol Vis Sci. 2011; 52(5):2306-2313. [PubMed: 21220551]

21. Porac C, Coren S. Sighting dominance and binocular rivalry. Am J Optom Physiol Optics. 1978; 55:208-213.

22. Borish, IM. Clinical Refraction. 3. Chicago: The Professional Press; 1970. p. 1192-1194.

23. Rutstein RP, Daum KM, Eskridge JB. Clinical characteristics of anomalous correspondence. Optom Vis Sci. 1989; 66(7):420-425. [PubMed: 2771327]

24. Blake R, O’Shea RP, Mueller TJ. Spatial zones of binocular rivalry in central and peripheral vision. Vis Neurosci. 1992; 8(5):469-478. [PubMed: 1586647]

25. Bronstad PM, Bowers AR, Albu A, Goldstein RB, Peli E. Driving with central visual field loss I: impact of central scotoma on response to hazards. JAMA Ophthalmology. 2013 Published online January 17, 2013. 10.1001/jamaophthalmol.2013.1443

26. Peli E, Yang J, Goldstein RB. Image invariance with changes in size: the role of peripheral contrast thresholds. J Opt Soc Am A. 1991; 8(11):1762-1774. [PubMed: 1744773]

27. Strasburger H, Harvey LO Jr, Rentschler I. Contrast thresholds for identification of numeric characters in direct and eccentric view. Percept Psychophys. 1991; 49(6):495-508. [PubMed: 1857623]

28. Gurnsey R, Roddy G, Chanab W. Crowding is size and eccentricity dependent. J Vis. 2011; 11(7): 15, 11-17. [PubMed: 21685401]

29. Jampolsky A. Characteristics of suppression in strabismus. Arch Ophthalmol. 1955; 54(5):683696.

30. Pratt-Johnson JA, Tillson G. Suppression in strabismus--an update. Br J Ophthalmol. 1984; 68(3): 174-178. [PubMed: 6696870]

31. Bagolini B. Anomalous correspondence: definition and diagnostic methods. Doc Ophthalmol. 1967; 23:346-398. [PubMed: 5583482]

32. Harley, RD.; Nelson, LB.; Olitsky, SE. Harley's Pediatric Ophthalmology. 5. Baltimore: Lippincott Williams \& Wilkins; 2005.

33. Seijas O, Gomez de Liano P, Gomez de Liano R, Roberts CJ, Piedrahita E, Diaz E. Ocular dominance diagnosis and its influence in monovision. Am J Ophthalmol. 2007; 144(2):209-216. [PubMed: 17533108] 
34. Neely DE, Sprunger DT. Nystagmus. Curr Opin Ophthalmol. 1999; 10(5):320-326. [PubMed: 10621545]

35. Woods RL, Apfelbaum HL, Peli E. DLP-based dichoptic vision test system. J Biomed Optics. 2010; 15(1):1-13.

36. Geisler W. Visual perception and the statistical properties of natural scenes. Annu Rev Psychol. 2008; 59:167-192. [PubMed: 17705683]

37. Pearson, K. On the Theory of Contingency and Its Relation to Association and Normal Correlation. Cambridge University Press; London: 1904.

38. Winer, BJ.; Brown, DR.; Michels, KM. Statistical Principles in Experimental Design. McGrawHill; New York: 1991.

39. Peli E. Vision multiplexing: An engineering approach to vision rehabilitation device development. Optom Vis Sci. 2001; 78(5):304-315. [PubMed: 11384008]

40. Bedell HE. Perception of a clear and stable visual world with congenital nystagmus. Optom Vis Sci. 2000; 77(11):573-581. [PubMed: 11138830]

41. American Association of Motor Vehicle Administrators \& The Motorists' Vision Committee of the American Optometric Optometric Association. Manual on drivers' vision test. Pittsburgh, PA: American Optometric Association; 1949. p. 32

42. Lippmann O, Corn AL, Lewis MC. Bioptic telescopic spectacles and driving performance: A study in Texas. J Vis Impair Blind. 1988; 82(5):182-187.

43. Doherty AL, Peli E, Luo G. Detection of hazards in driving videos with a monocular bioptic telescope (abstract). Optom Vis Sci. 2011; 88(Suppl):E-abstract 115022. 


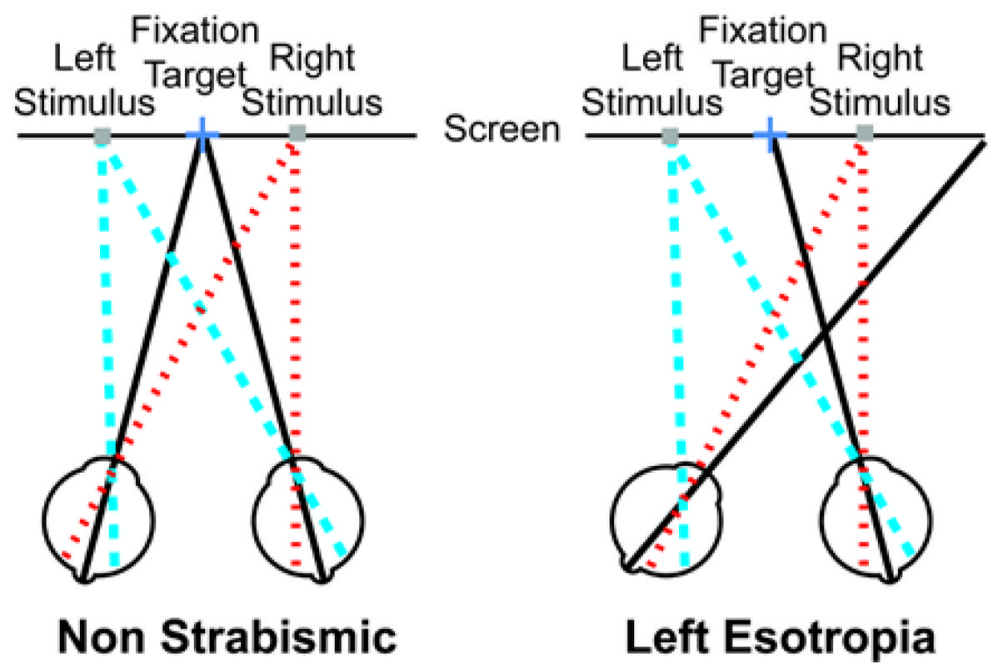

Figure 1.

An illustration (not to scale) of the retinal eccentricity of stimuli placed to the left (blue dashed lines) and right (red dotted lines) of fixation (black lines represent visual axes) for a non-strabismic patient and a left esotropic patient. The stimulus on the left falls on a more eccentric retinal area of the fellow (deviating) eye in the strabismic patient than it would in a patient without strabismus, as the eye turn is away from the stimulus (left eye turning to the right). The stimulus on the right falls on more central retina the fellow eye of the strabismic patient compared to the non-strabismic patient as the eye turn is toward the stimulus. 


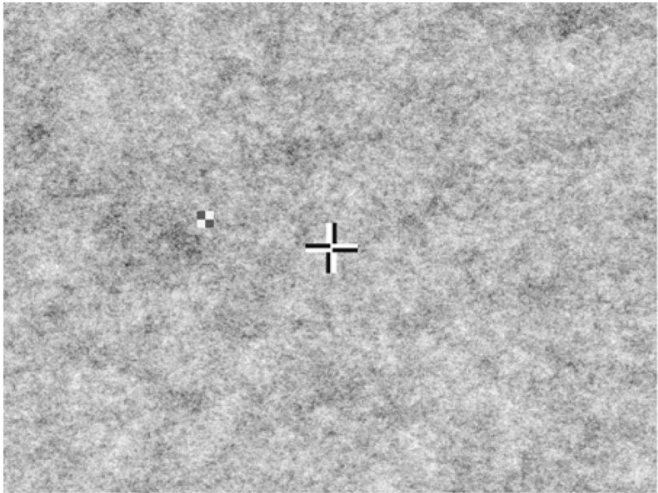

A

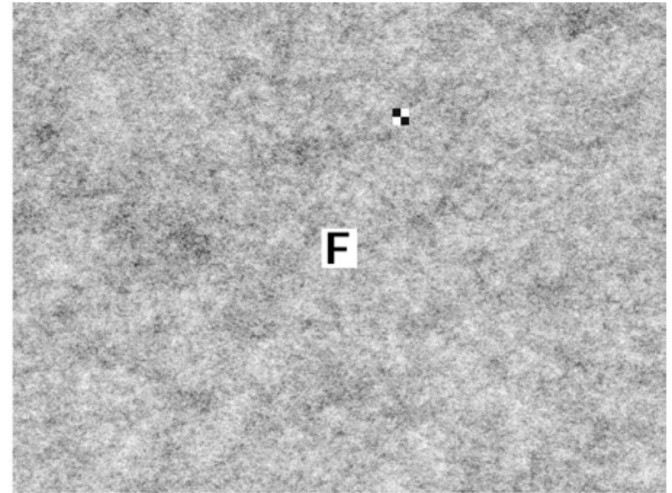

B

Figure 2.

The patterned background used in the study with: (A) $75 \%$ contrast checkerboard stimulus and passive fixation cross; and (B) 95\% contrast stimulus and active fixation letter. 


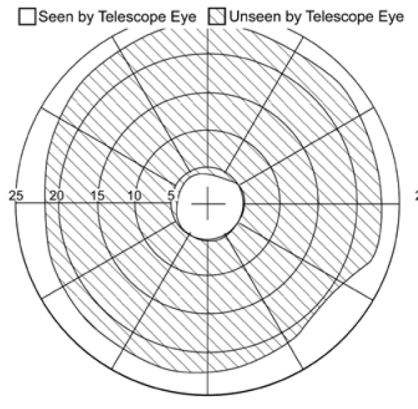

A

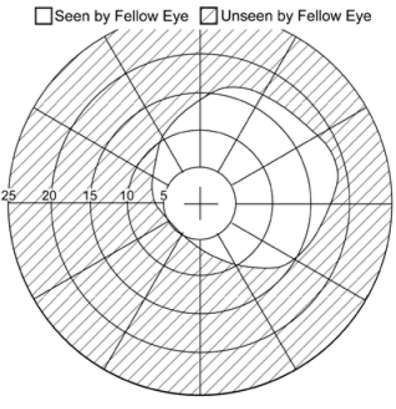

B

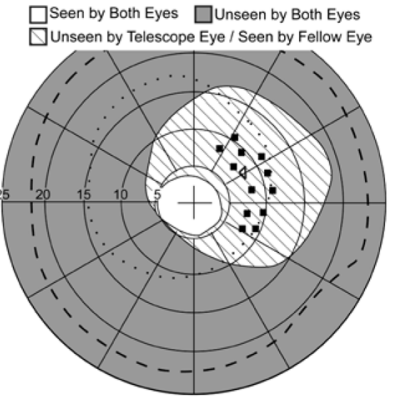

C

Figure 3.

An example of binocular visual field plots obtained for a right strabismic patient wearing a $3 x$ Galilean bioptic on the left eye. (A) Monocular viewing through the telescope without shutter lenses (fellow-eye patched) showing the ring scotoma of the telescope. (B) Asymmetric field of view through the fellow (right) eye shutter lens. Fixation and background were seen with both eyes while the perimetry stimulus was presented only to the fellow (non-telescope) eye. (C) Binocular viewing with the shutter lenses; fixation and background were seen by both eyes. The square symbols show the position of static stimuli (within the area of the telescope eye ring scotoma) presented to the temporal field of the fellow eye only. The left-facing triangle shows the position of the fixation catch trial static stimulus (in the ring scotoma) presented to the telescope eye only. The dashed line shows the outer boundary of the ring scotoma as measured in (A). The dotted line indicates the restriction of the telescope-eye shutter lens. 


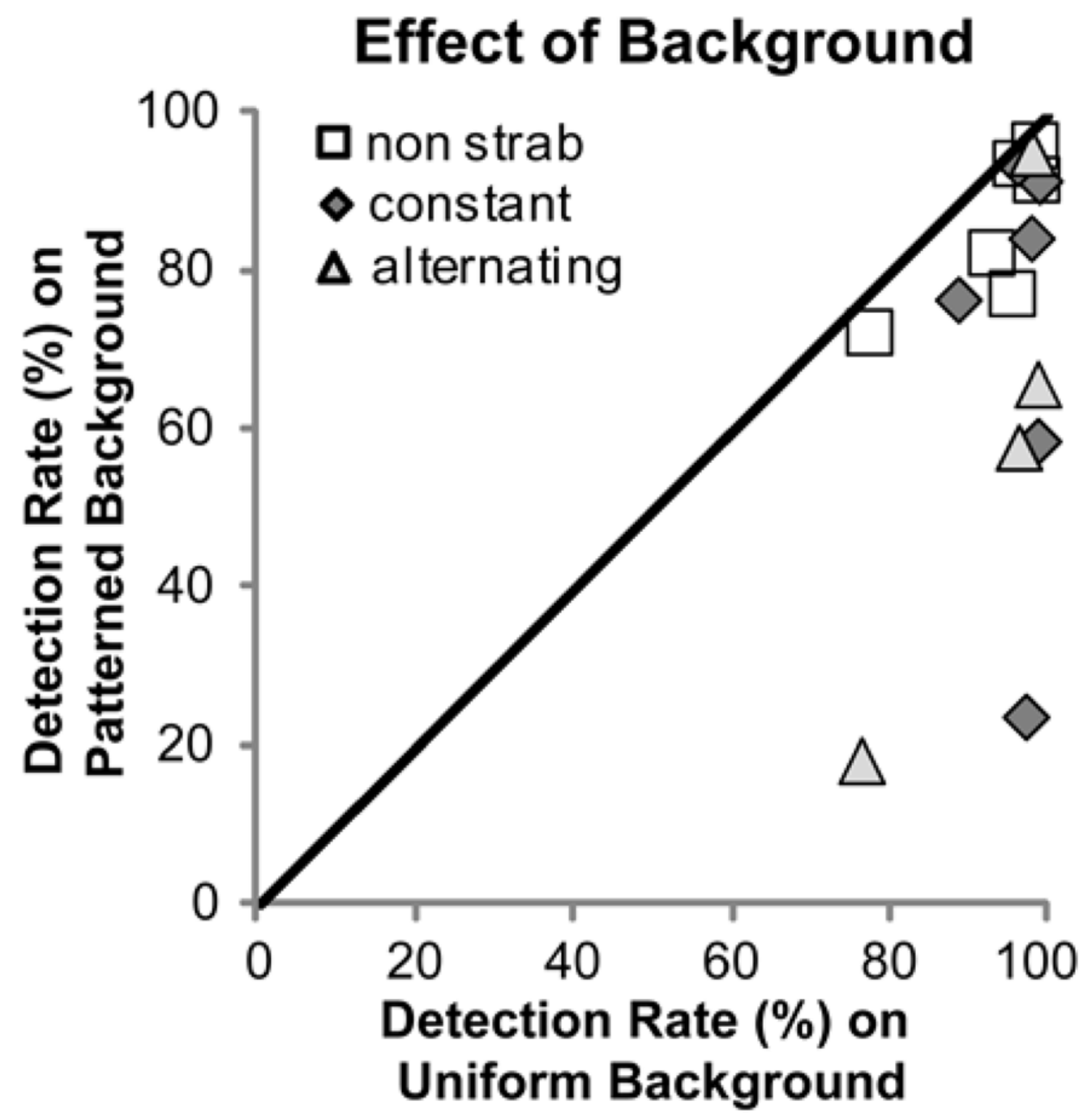

Figure 4.

Fellow-eye detection rates for each patient compared for the patterned and uniform backgrounds. For both strabismic and non-strabismic patients, detection rates were significantly lower on the patterned background (the majority of points are below the diagonal); however, the decrease was greater for strabismics (more points farther from the diagonal) than non-strabismics. 


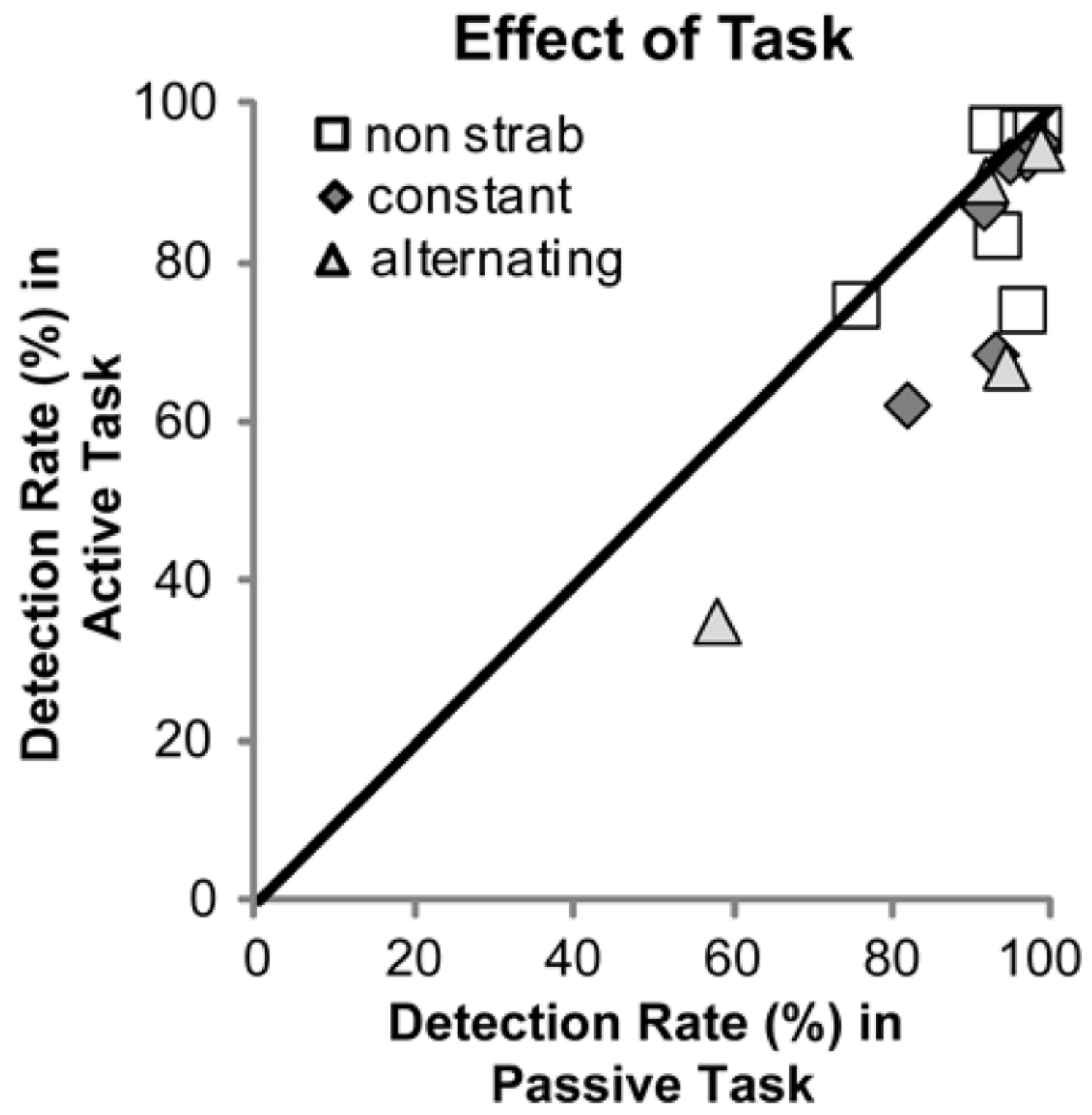

Figure 5.

Fellow-eye detection rates for each patient compared for the passive and active viewing tasks. There was a significant reduction in detection rates in the active fixation task. 


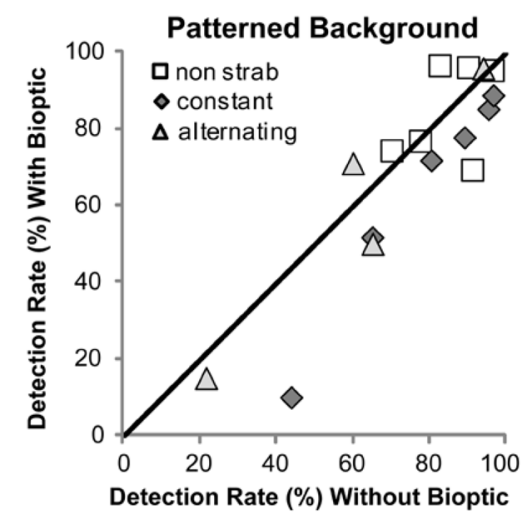

A

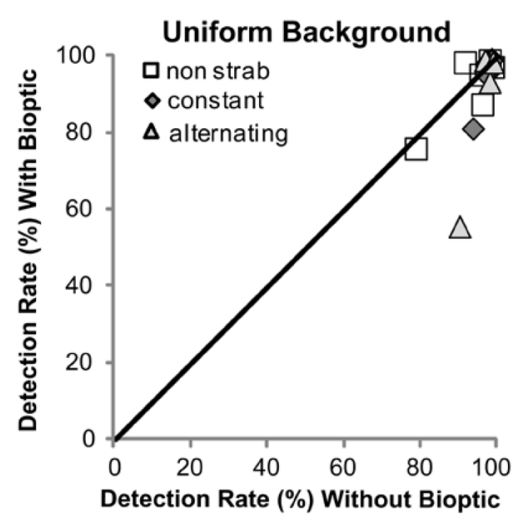

B

Figure 6.

Fellow eye detection rates for each patient comparing without and with the bioptic on (A) the patterned background and (B) the uniform background. On the patterned background, strabismic patients had significantly $(p=0.02)$ lower detection with the bioptic (majority of gray-filled symbols below the diagonal) while detection rates of non-strabismic patients (open squares) were not significantly different without and with the bioptic. On the uniform background, detection rates were similar (and high) without and with the bioptic for both groups. 


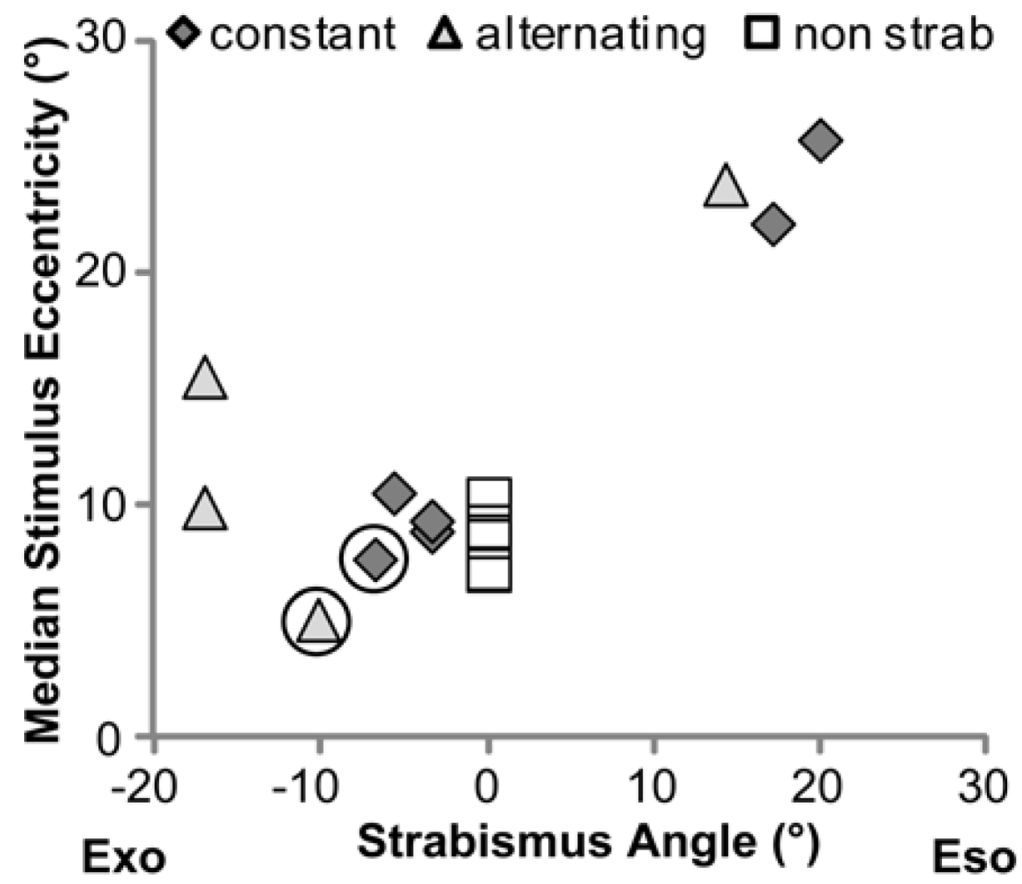

Figure 7.

Larger stimulus retinal eccentricities were associated with larger angle deviations in strabismic patients. For non-strabismic patients, the median stimulus eccentricity was $8^{\circ}$. The circled points are the 2 strabismic patients with a small difference in detection rates between the uniform and patterned backgrounds (see Figure 8). 


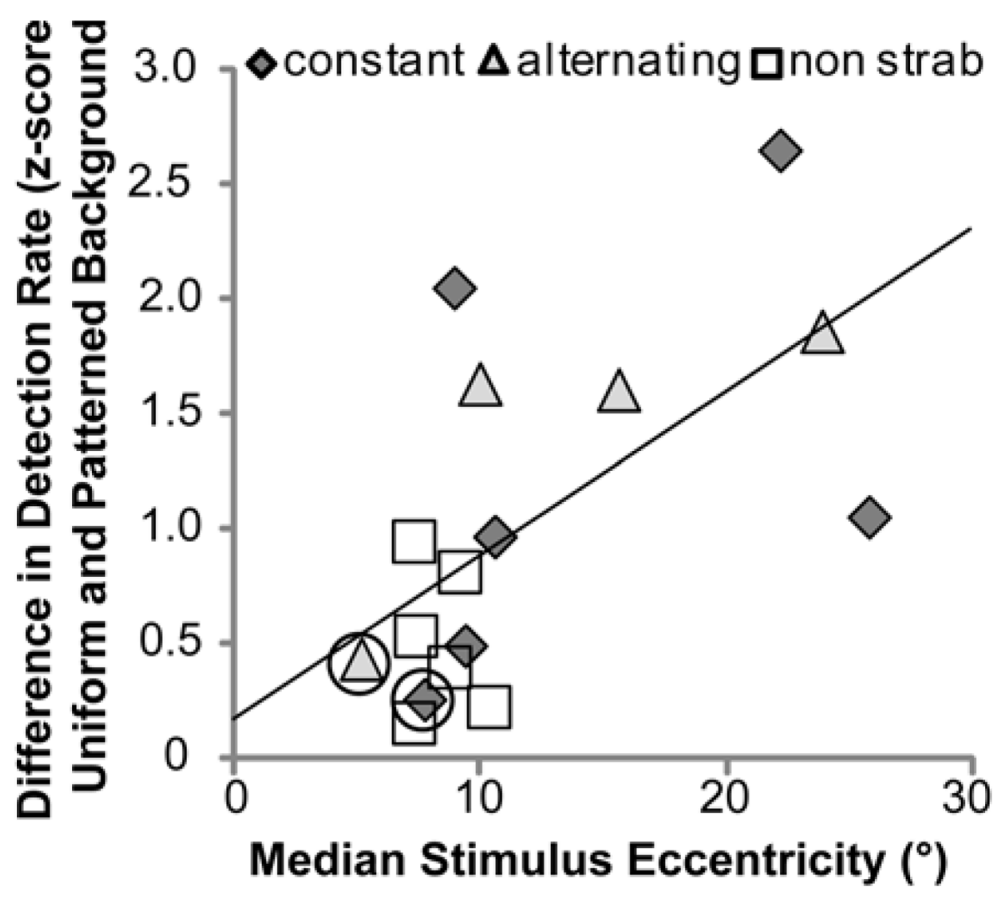

Figure 8.

Relationship between stimulus retinal eccentricity and the difference in fellow eye detection rates between the uniform and patterned backgrounds (data pooled across bioptic and task). Patients with larger stimulus eccentricities had larger reductions in fellow-eye detection rates $(\mathrm{rho}=0.621, \mathrm{p}=0.013)$. 


\section{Table 1}

Diagnoses for strabismic and non strabismic patients

\begin{tabular}{lcc}
\hline & Strabismic (n=10) & Non Strabismic (n=6) \\
Infantile Nystagmus Syndrome & 4 & 1 \\
Albinism (with nystagmus) & 4 & 1 \\
Ocular Albinism (with nystagmus) & 2 & 0 \\
Optic Atrophy & 0 & 3 \\
Rod Monochromat (with nystagmus) & 0 & 1 \\
\hline
\end{tabular}

Ophthalmic Physiol Opt. Author manuscript; available in PMC 2014 July 01. 
Table 2

Summary of vision measures for strabismic and non strabismic patients

\begin{tabular}{|c|c|c|c|c|}
\hline & Strabismic $(\mathrm{n}=10)$ & Non Strabismic $(\mathrm{n}=6)$ & $\begin{array}{c}\text { Test for Difference Between } \\
\text { Groups }\end{array}$ & p Value \\
\hline Male, no. (\%) & $9(90)$ & $4(66)$ & $x^{2}=1.3 \dagger$ & 0.247 \\
\hline Age, median (IQR), y & $38(32-47)$ & $39(33-44)$ & $\mathrm{U}=29.0 \hbar$ & 0.914 \\
\hline Nystagmus, no. (\%) & $10(100)$ & $3(50)$ & $x^{2}=6.2^{\dagger}$ & 0.013 \\
\hline $\begin{array}{l}\text { Telescope Eye VA }{ }^{\xi} \text { Without Bioptic, median } \\
\text { (IQR) }\end{array}$ & $6 / 28(6 / 26-6 / 30)$ & $6 / 25(6 / 22-6 / 29)$ & $\mathrm{U}=22.5 *$ & 0.413 \\
\hline Fellow Eye VA, ${ }^{\mathcal{s}}$ median (IQR) & $6 / 27(6 / 22-6 / 34)$ & $6 / 36(6 / 20-6 / 38)$ & $\mathrm{U}=22.5 \hbar$ & 0.415 \\
\hline Contrast Sensitivity, median (IQR) & $1.75(1.70-1.80)$ & $1.53(1.33-1.83)$ & $\mathrm{U}=20.0 \%$ & 0.277 \\
\hline Magnitude of strabismus, median (IQR), $\Delta$ & $22(10-30)$ & N/A & & \\
\hline 95\% contrast stimuli used, no. (\%) & $4(40)$ & $1(17)$ & $X^{2}=1.9+$ & 0.383 \\
\hline
\end{tabular}

$\mathrm{IQR}$, interquartile range,

${ }^{\dagger}$ By $x^{2}$ test,

tBy Mann Whitney U Test,

$\mathcal{S}_{\text {Visual acuity was measured and analyzed in logarithm of the minimum angle of resolution (logMAR) units; } \log \text { MAR values were converted to }}$ (integer) Snellen values for ease of interpretation in the table. 
Table 3

Median percent of fixation samples within $2^{\circ}$ and $4^{\circ}$ of the fixation target in the Nidek MP- 1 perimeter (only 14 patients completed this assessment)

\begin{tabular}{lcc}
\hline \multicolumn{2}{r}{ Patients with nystagmus (n=11) } & Patients without nystagmus (n=3) \\
Median \% Fixations within $2^{\circ}(\mathrm{IQR})$ & $96(96-98)$ \\
Dominant Eye & $60(58-70)$ & $97(87-98)$ \\
Fellow eye Eye & $77(46-84)$ & \\
Median \% Fixations within $4^{\circ}(\mathrm{IQR})$ & $100(100-100)$ \\
Dominant Eye & $94(91-96)$ & $100(94-100)$ \\
Fellow eye Eye & $96(84-98)$ &
\end{tabular}


Table 4

Results of repeated measures ANOVA.

\begin{tabular}{|c|c|c|c|c|}
\hline & Bioptic & Background & Task & Strabismus \\
\hline Bioptic (no bioptic vs bioptic) & $\begin{array}{c}\mathrm{F}_{(1,14)}=2.45 \\
\mathrm{p}=0.140 \\
92 \% \text { vs } 89 \%\end{array}$ & $\begin{array}{c}\mathrm{F}_{(1,14)}=0.043 \\
\mathrm{p}=0.838\end{array}$ & $\begin{array}{c}\mathrm{F}_{(1,14)}=2.10 \\
\mathrm{p}=0.170\end{array}$ & $\begin{array}{c}\mathrm{F}_{(1,14)}=2.35 \\
\mathrm{p}=0.147\end{array}$ \\
\hline Background (uniform vs patterned) & & $\begin{array}{c}F_{(1,14)}=28.90 \\
p<0.001 \\
96 \% \text { vs } 80 \%\end{array}$ & $\begin{array}{c}\mathrm{F}_{(1,14)}=0.095 \\
\mathrm{p}=0.762\end{array}$ & $\begin{array}{c}F_{(1,14)}=5.35 \\
p=0.036\end{array}$ \\
\hline Task (passive vs active) & & & $\begin{array}{c}F_{(1,14)}=10.78 \\
p=0.005 \\
93 \% \text { vs } 87 \%\end{array}$ & $\begin{array}{c}\mathrm{F}_{(1,14)}=1.57 \\
\mathrm{p}=0.230\end{array}$ \\
\hline Strabismus (nonstrabismus vs strabismus) & & & & $\begin{array}{c}\mathrm{F}_{(1,14)}=0.56 \\
\mathrm{p}=0.467 \\
92 \% \text { vs } 88 \%\end{array}$ \\
\hline
\end{tabular}

Shaded entries with bold font indicate significant effects 